\title{
Thermal Studies of Three-Dimensional Printing Using Pulsed Laser Heating
}

\author{
Yue Xiao, ${ }^{1}$ Zhihan Hong, ${ }^{2}$ Garret Coleman, ${ }^{3}$ Hongbo Zhao, ${ }^{1}$ Rongguang Liang, ${ }^{2}$ Pierre Lucas, ${ }^{3}$ and Qing Hao ${ }^{1, *}$
}

Laser-based three-dimensional (3D) printing of polymers is a promising technology in fabricating complicated structures for applications in bioengineering, optics and molding. Infrared (IR) laser-assisted thermal curing printing technique offers high controllability by heating up the sample locally. Compared with other techniques like ultraviolet (UV) curing, IR laser-assisted thermal curing avoids yellowing issue, which is a common problem in UV curing. Accurate thermal simulations of the polymer curing processes under the laser heat is crucial to the design and improvement of the printing apparatus. In this work, a multi-physics simulation is carried out to predict the temperature rise and the curing extent profile of polydimethylsiloxane (PDMS) heated by a periodic, pulsed laser. The simulation incorporates the coupling between the local heating and the curing extent change. The cured spot sizes are provided for the given duty cycle and laser pulse period. The exothermic enthalpy of PDMS during the curing process is also measured to improve the simulation accuracy. The simulation results are validated with experiments using a pulsed $2 \mu \mathrm{m}$ IR laser. This technique can control the polymer curing to achieve a minimum feature size of $\sim 20 \mu \mathrm{m}$. Various patterns are fabricated to demonstrate the flexibility of this technique.

Keywords: 3D printing; Thermal simulation; Laser-assist printing

Received 20 July 2018, Accepted 24 September 2018

DOI: $10.30919 /$ esmm5f103

\section{Introduction}

In recent years, additive manufacturing (AM) processes have experienced a rapid development in a variety of applications including fuel cells, ${ }^{1,2}$ bioengineering, ${ }^{3-6}$ and optical devices. ${ }^{7,8}$ As one of the $3 \mathrm{D}$ printing technologies, laser-based $\mathrm{AM}$ is a promising technique to process thermally-curable optical silicones and fabricate related devices, because the high power intensity from the laser can be achieved and contained in the small focal region to cure the material quickly and locally. ${ }^{7}$ Also, for thermally-curable silicones has the benefits of high-optical transmissivity and non-yellowing. In this technology, a continuous wave $(\mathrm{CW})$ or pulsed laser is employed to locally cure the polymer with heat. As an alternative method, ultra-violet (UV) curing of polymers has also been adopted by some commercial systems. ${ }^{9}$ However, the UV-processed polymers tend to age faster and appear to be yellow. ${ }^{8}$ These disadvantages limit the potential application of UV-based 3D printing in fabricating optical lenses for imaging applications.

Polymers like polydimethylsiloxane (PDMS) can be thermally cured by baking and retain their optical transmissivity for a rather long time. However, simple heating cannot offer the spatial resolution and controllability that is required for the fabrication of complicated 3D structures. In that respect, laser curing of thermally

\footnotetext{
${ }^{1}$ Department of Aerospace and Mechanical Engineering, University of Arizona, Tucson, AZ 85721 U.S.A

${ }^{2}$ College of Optical Sciences, University of Arizona, Tucson, AZ 85721 U.S.A

${ }^{3}$ Department of Material Science and Engineering, University of Arizona, Tucson, AZ 85721 U.S.A

*E-mail: qinghao@email.arizona.edu
}

curable polymers has several advantages, such as strong UV stability, non-yellowing, and high optical transmission. These features make the laser curing technology an ideal candidate for polymer-based 3D printing, especially in applications like optical imaging.

One of the important performance benchmarks for the 3D printing is the minimum feature size. Some applications like microfluidic devices and optical imaging require the minimum feature size to be on the scale of micrometers or even nanometers. In laser curing technology, reducing the feature size means to have better knowledge of the transient temperature profile and curing extent profile to minimize the heat spreading and thus reduce the achieved feature sizes. To address these concerns, tremendous research efforts have been dedicated to a better understanding the thermal transport process during laser heating. Some earlier theoretical studies focused on the temperature rise in monolayer materials, using the Fourier's law and Gaussian distribution of the laser intensity to provide an exact solution to the temperature profile of a slab heated by a laser beam..$^{10-12}$ Later studies further considered the temperature-dependent reflectivity and temperature-dependent absorption coefficient under the $\mathrm{CW}$ laser heating. ${ }^{13,14}$ For the pulsed laser, numerous thermal studies inside the heated material were carried out in the past. ${ }^{15-18}$ Particularly, the temperature profiles for different laser pulse duty cycles were studied using the finite element method. ${ }^{17}$ The difference in the heat accumulation caused by subsequent pulses and subsequent scans were also investigated. ${ }^{16}$ The thermal study of laser heating was also extended to multi-layered systems. ${ }^{19,20}$ Effects of different top-side boundary conditions on the thermal penetration depth within a multi-layered system were studied numerically. ${ }^{19}$ A quick estimation of the interface temperature variation was also developed for both $\mathrm{CW}$ and pulsed lasers. ${ }^{20}$ 
Despite numerous studies, the direct comparison between these theoretical studies and experiments are rare in the literature. More importantly, none of the above studies consider complex phase change processes such as the curing process of the polymer. In practice, such phase change is critical to the local temperature rise during the 3D printing and spatial resolution of the fabricated 3D structures. Keeping these in mind, it is important to develop a simulation technique that can couple both thermal transport and polymer curing under the laser heating process. Further comparison with experimental measurements is also required to validate the simulation results.

In this work, a comprehensive transient thermal simulation technique, which incorporates the polymer curing process, is carried out to predict the structure formation with a periodic pulsed laser. The simulations are further verified by experiments using a nanosecond pulsed infrared (IR) laser. Without losing the generality, PDMS is selected as the studied polymer for its high transparency, good bio-compatibility ${ }^{22}$ and a wide operating temperature range from $228 \mathrm{~K}$ to at least $473 \mathrm{~K}^{23,24}$ Under the laser heat, PDMS is cured through an exothermic cross-linking process. To improve the accuracy of thermal modeling, the exothermic heat generated in the curing process is measured by Differential Scanning Calorimetry (DSC) and then adopted in the simulation. The curing extent of PDMS is incorporated into the thermal simulation, as described by the Arrhenius equation. ${ }^{25}$ The thermal simulation renders the local temperature evolution within the PDMS and the size of cured PDMS spots, the latter of which is particularly important to determining the spatial resolution (i.e., minimum feature size) of this 3D printing technique. In experiments, dot patterns are fabricated to verify the simulation results. Other patterns are also fabricated under the guidance of the thermal simulation, with a minimum feature size of $\sim 20 \mu \mathrm{m}$. The simulation technique presented here can be critical to the future development of laser-assisted thermal curing 3D printing technology.

\section{Methods Physical Model for COMSOL Simulations}

All simulations are carried out with COMSOL Multiphysics software package, with the curing extent study input as customized physics to be coupled with the thermal analysis of the basic package. The theoretical model depicts the scenario where a laser beam is incident onto a surface of a PDMS block. This model solves for the temperature profile and curing extent profile within the computational domain. For a Cartesian coordinate system, the conventional heat diffusion equation ${ }^{26}$ can be adopted:

$$
\rho c_{p} \frac{\partial T}{\partial t}=k\left(\frac{\partial^{2} T}{\partial \chi^{2}}+\frac{\partial^{2} T}{\partial y^{2}}+\frac{\partial^{2} T}{\partial z^{2}}\right)+Q^{\prime \prime \prime},
$$

where $k, T, Q$ '” $\rho$ and $c_{p}$ are the thermal conductivity, temperature, volumetric heat generation rate, density and specific heat, respectively. In this heat diffusion equation, $k$ and $c_{p}$ are approximated as constants because the thermal properties of PDMS are virtually unchanged before and after the curing process. ${ }^{24}$ The heat diffusion equation is further coupled with the Arrhenius equation ${ }^{25}$ with $2^{\text {nd }}$-order curing kinetics to predict the curing extent rate:

$$
\frac{\partial \alpha}{\partial t}=k_{0} e^{-\frac{E \alpha}{R T}}(1-\alpha)^{n} \alpha^{m}
$$

where $\alpha, k_{0}, E_{a}$ and $R$ represent the curing extent as a percentage value, pre-exponential factor, activation energy and universal gas constant, respectively. The pre-exponential factor $k_{0}$, representing the frequency of collisions in the correct orientation, is a constant for each chemical reaction. Here $n$ and $m$ are two fitted coefficients. In Eq. (2), all parameters are adopted from a $2^{\text {nd }}$-order model for PDMS by setting $n=2$ and $m=0$ so that the term $\alpha^{m}$ vanishes. ${ }^{27}$ For other thermally curable polymers, these fitted coefficients can be acquired from DSC measurements, following the ASTM procedure E2070-08 standard.

In simulations, the volumetric heat generation rate $Q$ "'consists of the local curing-reaction heat generation $q_{\text {cure }}(\partial \alpha / \partial t)$ and absorbed laser heat $q_{\text {laser }}$, both of which are evaluated per unit volume and unit time. The curing reaction heat generation is induced by the PDMS cross-linking process during laser curing. When the curing process has completed, $\partial \alpha / \partial t$ will reduced to zero and $Q$ "' only contains $q_{\text {laser }}$

To compute $q_{\text {cure }}$ for the variation of curing extent $\alpha$, the expression $q_{\text {cure }}=-\rho \Delta h$ is used. A constant density is assumed for PDMS, and $\Delta h$ is the enthalpy change per unit mass. For PDMS, the existing studies for $\Delta h$ is rare and its value is often treated as zero for simplification. ${ }^{6}$ To improve the accuracy of the simulation, $\Delta h$ is measured via a heat flow DSC (DSC1, Mettler-Toledo) in this work. The PDMS samples are heated up at a rate of $10 \mathrm{~K} / \mathrm{min}$, and $\Delta h$ can be calculated by measuring the change of heat flow released by the sample. Fig. 1 shows the heat flow $(\dot{Q})$ variation with the increase of the temperature, the heat flow is denoted by the solid black line. A baseline (solid blue line) representing the case without the heat generation is extrapolated from the base heat flow. By integrating the area between these two lines, the total heat transfer during the heating process (blue area in Fig. 1) can be measured. Then $\Delta h$ can be calculated by

$$
\Delta h=\frac{1}{m} \int_{T_{1}}^{T_{2}} \frac{\Delta \dot{Q}}{\left(\frac{\Delta T}{\Delta t}\right)} d T,
$$

where $m$ is the sample mass, $\Delta \dot{Q}$ is the heat flow difference between the signal and baseline curves, and $(\Delta T / \Delta t)$ is the temperature variation rate $(10 \mathrm{~K} / \mathrm{min})$. Temperatures $T_{1}$ and $T_{2}$, as indicated in Fig. 1, are those when the reaction starts and ends, respectively.

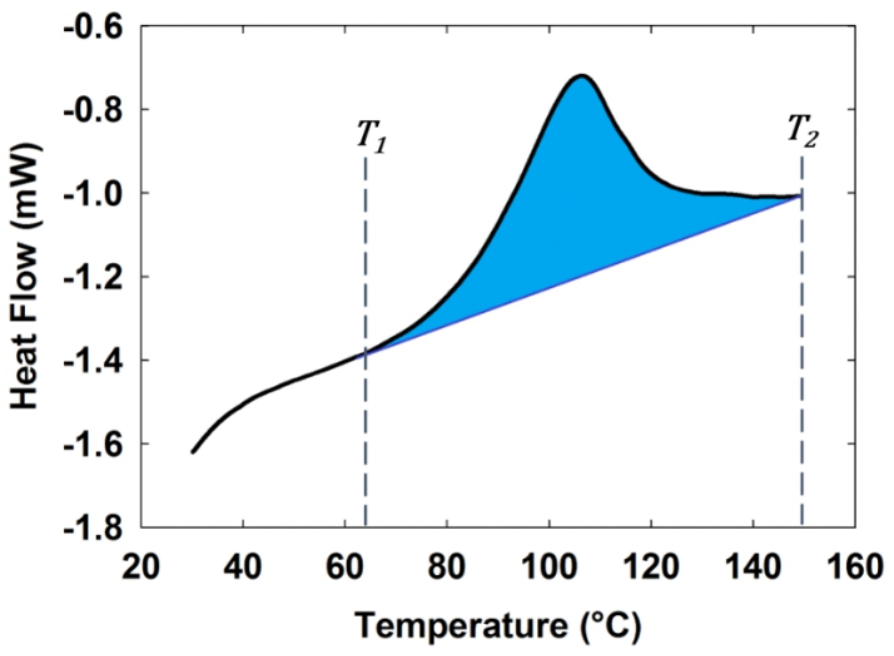

Fig. 1 DSC-measured heat flow. 
For volumetric heat generation rate $q_{\text {laser }}$ due to laser absorption, the employed laser is assumed to be focused onto a spot on the surface of the PDMS. Fig. 2 illustrates the basic geometry of this laser-heating setup, where $P_{0}$ and $\theta$ represent the laser output power and focus cone angle, respectively.

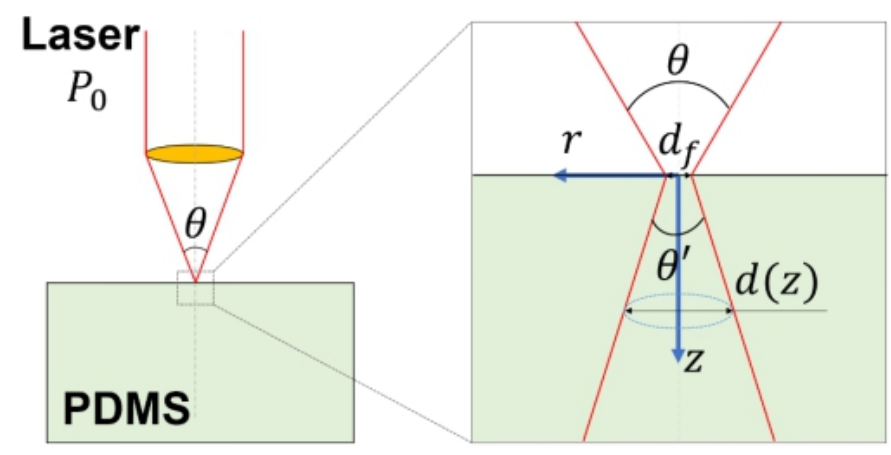

Fig. 2 Illustration of laser heating simulation model.

On the PDMS surface, $d_{f}$ is the diameter of the laser spot. The refractive index, $n_{P D M S}$, is $\sim 1.4$ for PDMS. ${ }^{28}$ The cone angle inside the PDMS is denoted as $\theta^{\prime}$, which can be computed by $\sin \left(\theta^{\prime} / 2\right)=\sin (\theta / 2) / n_{P D M S}$ using Snell's law. The positive z-direction is set to be starting from the PDMS surface. The diameter of the laser inside the PDMS $d(z)$ will be approximated in the form of

$$
d(z)=d_{f}+2 z \tan \frac{\theta^{\prime}}{2}
$$

Then the laser-absorption power density in the PDMS is calculated using Beer-Lambert law. ${ }^{29}$ The laser power at a plane with depth $z$ is

$$
P(z, t)=\gamma P_{0}(t) e^{-\kappa z},
$$

where $\gamma$ represents the transmissivity of the air-PDMS interface and $\kappa$ is the approximately constant absorption coefficient of PDMS. Here $P_{0}(t)$ represents the instantaneous laser power at the air-PMDS interface. A detailed description on the pulse laser will be given in the experimental setup part. With Eq. (5), the total absorbed power by PDMS from the top surface to a plane with a depth $z$ is

$$
P_{t o t}(z, t)=\gamma P_{0}(t) \cdot\left(1-e^{-k z}\right),
$$

and the absorbed power by a thin layer of PDMS at depth $\mathrm{z}$ with a thickness $d z$ is

$$
d P_{t o t}(z, t)=\gamma P_{0}(t) \kappa e^{-\kappa z} d z
$$

Finally, the geometric Gaussian profile of the laser beam is further considered in the simulation. In a plane perpendicular to the laser propagation direction, the laser intensity is proportional to $\exp \left(-2 r^{2} / b^{2}\right)$, where $r$ represents the radial distance from the axial line of the laser beam, and $b$ is the radius where the laser intensity drops to $e^{-2}$ of the intensity at $r=0$. Following this, the heat flux due to laser radiation is

$$
q^{\prime \prime}(r, z, t)=\frac{2 P_{t o t}(z, t)}{\pi b^{2}} \exp \left(-2 r^{2} / b^{2}\right) .
$$

With $b=d / 2$, the volumetric heat generation rate due to laser absorption is thus

$$
q_{\text {laser }}(r, z, t)=\frac{\partial q^{\prime \prime}(r, z, t)}{\partial z} \approx \frac{8 \kappa}{\pi d^{2}} \gamma P_{0}(t) \exp \left(-\frac{8 r^{2}}{d^{2}}-\kappa z\right),
$$

where the small variation of $b(z)$ within the $d z$ thickness is neglected.

Table I provides all PDMS-related thermal properties and geometry parameters, which can be used to determine $Q$ "' in Eq. (1). The temperature profile is first predicted with Eq. (1) and input into Eq. (2) to render the curing extent rate $\partial \alpha / \partial t$. Finally, $\partial \alpha / \partial t$ is used to update $Q$ " for the temperature prediction of the next time step. Above procedures are repeated to yield the temperature evolution and curing extent history within the whole computation domain. Based on the laser intensity profile indicated by Eq. (8), the cured PDMS will have a circular shape if observed from above.

Table I. Parameters and material properties.

\begin{tabular}{lcc}
\hline Parameter & Symbol & Value \\
\hline PDMS, thermal conductivity & $k$ & $0.15 \mathrm{~W} /(\mathrm{m} \cdot \mathrm{K})$ \\
PDMS, specific heat & $c_{p}$ & $1540 \mathrm{~J} /(\mathrm{kg} \cdot \mathrm{K})$ \\
PDMS, density & $\rho$ & $1030 \mathrm{~kg} / \mathrm{m}^{3}$ \\
PDMS, refractive index & $n_{P D M S}$ & 1.4 \\
Frequency factor & $K_{0}$ & $1.0225 \times 10^{13} 1 / \mathrm{s}$ \\
Activation energy & $E_{a}$ & $10.9 \times 10^{5} \mathrm{~J}$ \\
Universal gas constant & $R$ & $8.3140 \mathrm{~J} /(\mathrm{mol} \cdot \mathrm{K})$ \\
Fitted coefficient & $n$ & 2.0 \\
Enthalpy generation per unit mass & $\Delta h$ & $-20.79 \mathrm{~J} / \mathrm{g}$ \\
Absorption coefficient & $\kappa$ & $5.8 \times 10^{-2} 1 / \mathrm{mm}$ \\
Transmissivity of laser focusing lens & $\gamma$ & 0.96 \\
Focus angle & $\theta$ & $9^{\circ}$ \\
Focal spot diameter & $d_{f}$ & $0.05 \mathrm{~mm}$ \\
Top-of-PDMSto focal point distance & $L_{f}$ & $0 \mathrm{~mm}$ \\
Top surface heat transfer coefficient & $h_{t o p}$ & $18 \mathrm{~W} / \mathrm{m}^{2} \cdot \mathrm{K}$ \\
\hline
\end{tabular}

As the boundary conditions, the top surface has a convective heat transfer coefficient $h_{\text {top }}$ of $18 \mathrm{~W} / \mathrm{m}^{2} \cdot \mathrm{K}$ to account for the convection heat loss to the ambient. ${ }^{30}$ The bottom and the sidewall of the simulated PDMS domain are assumed to be at $300 \mathrm{~K}$ because these boundaries are far away from the heating spot.

\section{Experimental Setup}

For the verification purpose, the experiment is designed to match the simulation conditions. In the experiment, the optical silicone Dow Corning Sylgard-184 PDMS is used. The curing agent (cross-linker) and PDMS pre-polymer (oligomer) are mixed in a 1:10 weight ratio. The mixed PDMS is then spin-coated onto a microscope slide with
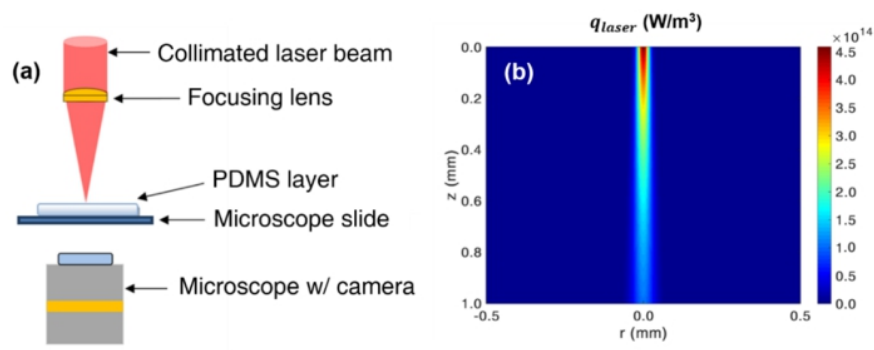

Fig. 3 (a) Illustration of the experimental setup. (b) $\boldsymbol{q}_{\text {laser }}$ distribution inside PDMS, within a plane cut along the axis of the laser beam.

A Q-switched fiber laser (AP-QS1-MOD, AdValue Photonics Inc.) is employed to achieve localized rapid curing. The position of 
the laser is adjusted so that the focus spot is at the top surface of the pre-cured polymer. The collimated laser beam, with a diameter of $8 \mathrm{~mm}$, passes through a plano-convex focusing lens with a $50 \mathrm{~mm}$ effective focal diameter. On the PDMS surface, the actual focal spot diameter is measured by a beam profiler to be $50 \mu \mathrm{m}$. The pulsed IR laser has a wavelength of $1.95 \pm 0.05 \mu \mathrm{m}$ and a frequency of $30 \mathrm{kHz}$. Other related parameters to determine $P_{0}(t)$ are given in Table II. With these parameters, $q_{\text {laser }}(r, z, t)$ can be calculated with Eq. (9). Fig. $3 \mathrm{~b}$ shows the $q_{\text {laser }}$ distribution inside the PDMS within the period of a laser pulse. The maximum $q_{\text {laser }}$ can be above $4.5 \times 10^{14} \mathrm{~W} / \mathrm{m}^{3}$. A fast decay of $q_{\text {laser }}$ along the z-direction can be observed from Fig. $3 \mathrm{~b}$. To obtain the relationship between the laser-heating time and the diameter of the laser-cured PDMS spot, an increased pulse number is implemented. Throughout the curing process, the microscope camera is used to monitor the spot diameter. Pulse numbers ranging from 150 to $>1000$ are tested to compare to the thermal simulation results.

Table II. Laser parameters.

\begin{tabular}{lc}
\hline Parameter & Value \\
\hline Selected average power & $8.5 \mathrm{~W}$ \\
Max. average power & $>10.0 \mathrm{~W}$ \\
Max. pulse energy & $>333 \mu \mathrm{J}$ \\
Pulse width & $30 \pm 5 \mathrm{~ns}$ \\
Frequency & $30 \mathrm{kHz}$ \\
Center wavelength & $1.95 \pm 0.05 \mu \mathrm{m}$ \\
\hline
\end{tabular}

To print more complicated patterns, the PDMS-coated microscope slide is placed on top of a two-axle motorized translation stage (PT1-Z8, Thorlabs). By controlling the stage movement and the laser heating time, various $2 \mathrm{D}$ and $3 \mathrm{D}$ patterns can be fabricated with this setup. The translation stage has $29 \mathrm{~nm}$ resolution for the movement, $0.05 \mu \mathrm{m}$ in minimum incremental movement, and $0.2 \mu \mathrm{m}$ in minimum repeatable incremental movement.

\section{Results and discussion Simulation results}

The temperature evolution and curing extent increase can be estimated from the proposed thermal analysis model. Fig. 4 presents the change of temperature $T$ at the laser-focused point (i.e., $r=0, z=$ 0 ), where temperature is consistently the highest within the heated PDMS. It can be observed that the temperature can go up to $1000 \mathrm{~K}$ when the heating time is at around $10 \mathrm{~ms}$. Generally, the degradation rate of PDMS increases radically at above $900 \mathrm{~K}$ with a fast heating rate. ${ }^{31}$ Experiments have shown the formation of methane at a high heating rate, due to the $\mathrm{Si}-\mathrm{CH}_{3}$ bond cleavage and hydrogen abstraction. ${ }^{23,32}$ Once the degradation starts, this proposed simulation model starts to become invalid because this model does not consider the generation of by-products. However, in practice, the heating time of a single dot will not go beyond $6 \mathrm{~ms}$, which is marked with a brown grid in Fig. 4. It is observed from Fig. 4 that the center point temperature will not go beyond $800 \mathrm{~K}$ at $6 \mathrm{~ms}$. Also, it is predicted that the majority of the PDMS will remain at a lower temperature because the laser power density is much weaker at regions away from the center point. Additionally, a sawtooth shape of the temperature change can be observed from the inset of Fig. 4. This sawtooth shape of the temperature variation reflects the results of using the pulsed laser heating. Within each $35 \mathrm{~ns}$ long laser pulse, the center-point temperature will increase quickly, then decrease during the unheated period. The sawtooth shape also agrees with other thermal studies on the pulsed laser heating. ${ }^{16}$

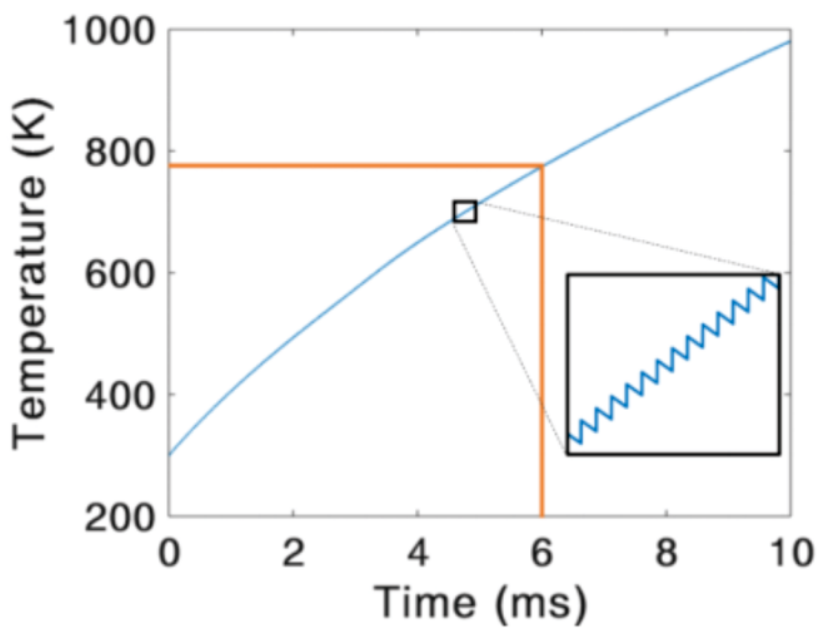

Fig. 4 The evolution of $\mathrm{T}(r=0, z=0, t)$

The curing extent information can be obtained from the simulation model as well. The curing extent of $99 \%$ is adopted as the threshold of whether a cured spot has formed. Fig. 5a shows the diameter of a cured PDMS spot at the $\mathrm{z}=0$ plane after 180 laser heating pulses, which is $\sim 28 \mu \mathrm{m}$. Using different heating times in the simulation, the relation between the cured-spot diameter and laser pulse number can be acquired.

The exact geometry of a cured PDMS spot can be extracted from the simulation. Fig. $5 \mathrm{~b}$ provides a cross-sectional view of the curing extent distribution. It can be observed that the cured volume has a roughly half-ellipsoid shape. With longer heating time, the depth and the radius of the ellipsoid both expand. The depth-toradius ratio will also increase with longer heating time, due to the highly concentrated laser-beam intensity within the laser beam.
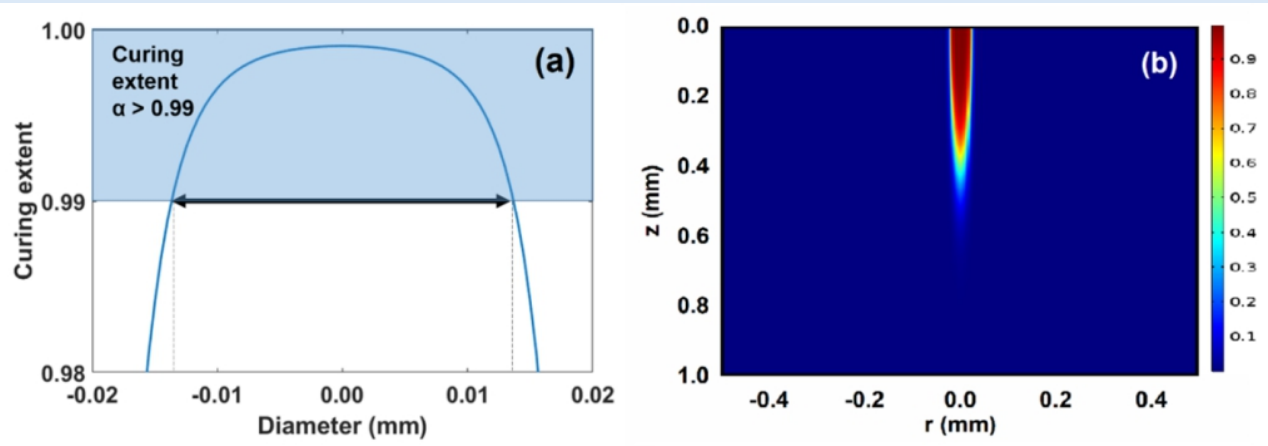

Fig. 5 (a) Curing extent within the $\mathrm{z}=0$ plane after 180 pulses. (b) Curing extent profile at 180 pulses, within a plane cut along the axis of the laser beam. 


\section{Comparisonto the experiment for single-spot printing}

As one of the most important predictions, the cured-spot diameter within the $\mathrm{z}=0$ plane is examined experimentally. Fig. 6 shows the bottom-view image of a cured spot taken by the integrated camera installed under the microscope slide. This image shows a circular shape of the cured region, as indicated in Eq. (9). The same measurements are carried out for different numbers of laser pulses. Fig. 7 compares the predicted and measured diameter of the cured region at the $\mathrm{z}=0$ plane, with respect to the laser pulse number. The simulation results agree well with the experimental data under 800 pulses. Above 800 pulses, a larger deviation is observed between the simulation and the experiment. This deviation can be explained by the PDMS degradation at higher temperatures. In the simulation with more than 800 pulses (i.e., $26 \mathrm{~ms}$ heating time), the center point temperature will reach $\sim 1200 \mathrm{~K}$, which is far beyond the stability range of PDMS. As anticipated, the simulation will become inaccurate at such a high temperature. When the laser pulse number is minimized to fabricate fine patterns, the simulation is always accurate to guide the experiments.

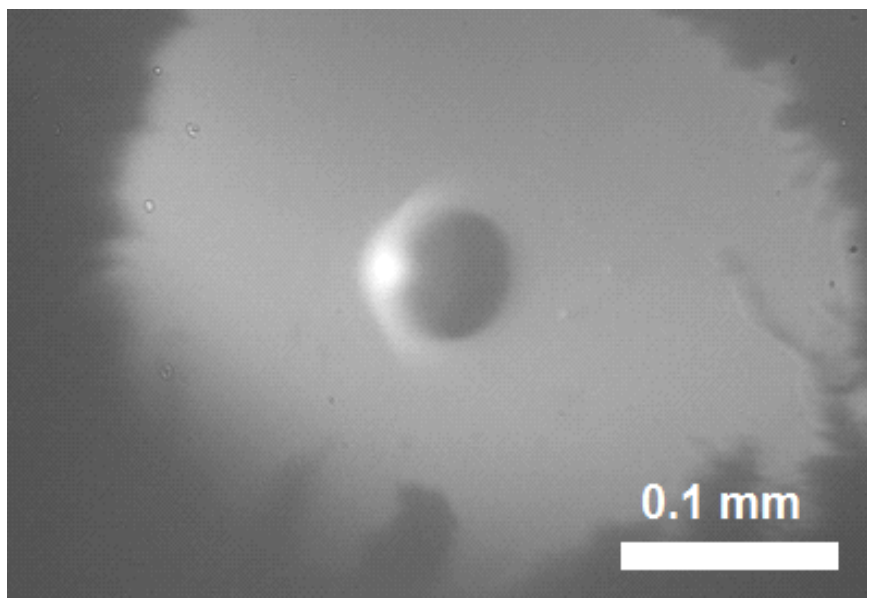

Fig. 6 Bottom-view optical microscope picture of a printed PDMS spot.

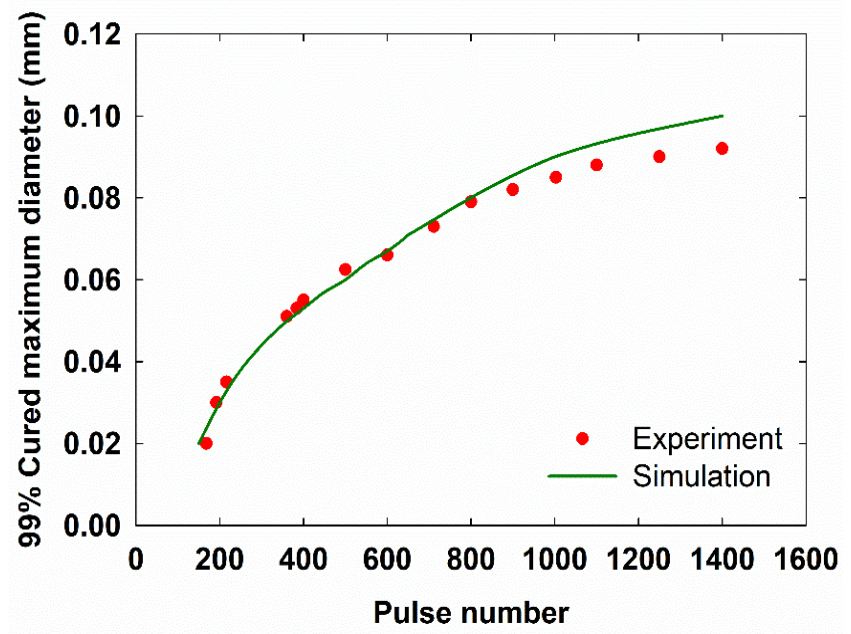

Fig. 7 Comparison between the simulation predictions and experiments for the cured-spot diameter at $\mathrm{z}=0$.

\section{Pattern printing}

Beyond single-spot printing, pattern printings such as wires and surfaces are performed using the alternative experimental setup with the translation stage. Fig. 8 shows a laser-printed line (top-right corner) and surface topography measured using white-light interferometry (Zygo Newview 8300). A scan route, as illustrated in Fig. 8 , is set in the white-light interferometry to extract the surface undulation of the printed line. The PDMS lines are printed as a row of dots, with 156 laser pulses per dot. These cured PDMS dots are located on the surface of PDMS, and the average pitch is around $20 \mu \mathrm{m}$. In Fig. 8, it can be observed that a relatively small bump is formed at center of each dot, which becomes the major contribution of the surface roughness. With $\sim 20 \mu \mathrm{m}$ dot pitch, the root-meansquare (RMS) surface roughness is measured at $0.07 \mu \mathrm{m}$ by whitelight interferometry and the z-direction bottom-to-top distance within each pitch is $\sim 0.2 \mu \mathrm{m}$.

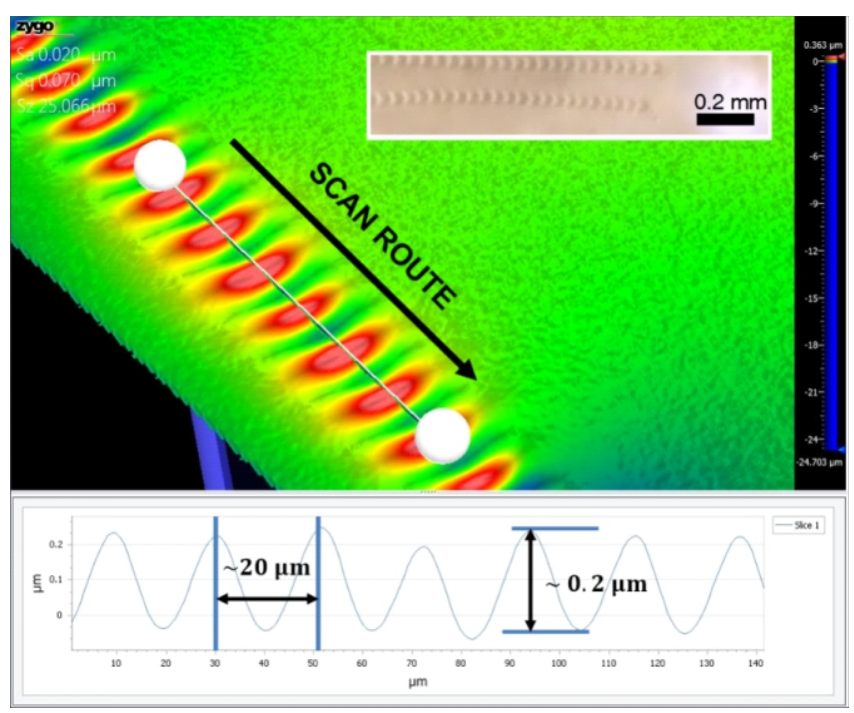

Fig. 8 White-light interferometry picture of a printed line consisting of a row of cured dots.

Fig. 9 presents a printed PDMS layer consisting of an array of dots, to demonstrate the potential to create 3D stacked structures layer by layer with an additive manufacturing process. Similarly, the subsequent pulse scheme with 144 pulses are used to print each dot. Fig. 9a is the microscopic image taken from the top of the printed layer. Fig. $9 \mathrm{~b}$ shows the measured results on the surface topography. The scan route is marked in Fig. $9 \mathrm{~b}$ as well. The z-direction bottomto-top distance within each pitch is reduced to $\sim 0.1 \mu \mathrm{m}$.

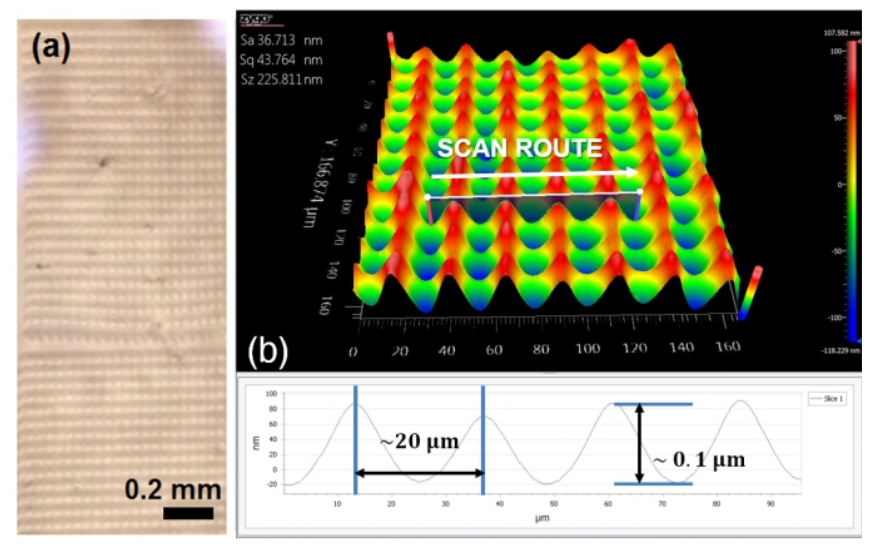

Fig. 9 (a) Optical image and (b) measured surface topography of a printed PDMS layer. 


\section{Conclusions}

In this work, a Fourier's law-based transient thermal simulation has been performed to guide the polymer-based $3 \mathrm{D}$ printing with pulsed IR laser heating. As an improvement to existing studies, ${ }^{15-18}$ the laser heating and polymer curing are fully coupled in the thermal simulation that is further compared to the experiment results. The temperature history and cured-spot diameter are predicted, and the latter is verified with the single-dot printing experiments with PDMS. The prediction agrees well with the experiments when the number of laser pulses is less than $\sim 800$, which is sufficient in practice. To accurately determine the volumetric heat generation rate in the thermal analysis, the exothermic enthalpy change for PDMS curing is measured via DSC. More complicated patterns, from a single line to an array of dots, are fabricated to show the flexibility of the proposed technology in fabricating sophisticated structures. Such laser-based $3 \mathrm{D}$ printing can be widely used to fabricate microscale optical devices, porous structures, and microfluidic devices.

\section{Conflict of interest}

There are no conflicts to declare.

\section{Acknowledgements}

This work is supported by National Science Foundation CAREER Award under Award No. CBET-1651840.

\section{References}

1. J. You, R. J. Preen, L. Bull, J. Greenman and I. Ieropoulos, Sustain. Energy Technol. Assessments, 2017, 19, 94-101.

2. S. C. Ligon, R. Liska, J. Stampfl, M. Gurr and R. Mülhaupt, Chem. Rev., 2017, 117, 10212-10290.

3. H. N. Chia and B. M. Wu, J. Biol. Eng., 2015, 9, 4.

4. S. Vijayavenkataraman, J. Fuh and W. Lu, Bioengineering, 2017, 4, 63.

5. R. Xiong, Z. Zhang, W. Chai, Y. Huang and D. B. Chrisey, Biofabrication, 2015, 7, 45011.

6. H. Selvaraj, B. Tan and K. Venkatakrishnan, J. Micromechanics Microengineering, 2011, 21, 75018.

7. Z. Hong and R. Liang, Sci. Rep., 2017, 7, 7145.

8. A. Heinrich, M. Rank, P. Maillard, A. Suckow, Y. Bauckhage, P. Rößler, J. Lang, F. Shariff and S. Pekrul, Adv. Opt. Technol., 2016, 5, 293-301.

9. Bridget Butler O'Neal, Comparing LUXeXceL's Printoptical Technology to Transparent Polyjet, SLA \&amp; MJM 3D Printing | 3DPrint.com | The
Voice of 3D Printing / Additive Manufacturing, https://3dprint.com

/49218/luxexcel-printoptical-clear/, (accessed 27 March 2018).

10. K. Brugger, J. Appl. Phys., 1972, 43, 577-583.

11. M. Lax, J. Appl. Phys., 1977, 48, 3919-3924.

12. J. F. Ready, J. Appl. Phys., 1965, 36, 462-468.

13. J. Mazumder and W. M. Steen, J. Appl. Phys., 1980, 51, 941-947.

14. E. Liarokapis and Y. S. Raptis, J. Appl. Phys., 1985, 57, 5123-5126.

15. M. Mirkov, E. A. Sherr, R. A. Sierra, J. R. Lloyd and E. Tanghetti, $J$. Appl. Phys., 2006, 99, 114701.

16. R. Weber, T. Graf, C. Freitag, A. Feuer, T. Kononenko and V. I. Konov, Opt. Express, 2017, 25, 3966.

17. S. Z. Shuja, B. S. Yilbas and S. Z. Shazli, Heat Mass Transf. und Stoffuebertragung, 2007, 43, 949-955.

18. E. G. Gamaly, A. V. Rode and B. Luther-Davies, J. Appl. Phys., 1999, 85, 4213-4221.

19. J. L. Braun and P. E. Hopkins, J. Appl. Phys., 2017, 121, 175107.

20. Y. Xu, R. Wang, S. Ma, L. Zhou, Y. R. Shen and C. Tian, J. Appl. Phys., 2018, 123, 25301.

21. J. L. Wilbur, R. J. Jackman, G. M. Whitesides, E. L. Cheung, L. K. Lee and M. G. Prentiss, Chem. Mater., 1996, 8, 1380-1385.

22. R. W. Hergenrother, Y. Xue-Hai and S. L. Cooper, Biomaterials, 1994, 15, 635-640.

23. G. Camino, S. . Lomakin and M. Lageard, Polymer (Guildf)., 2002, 43, 2011-2015.

24. D. Corning, Prod. Datasheet, 2013, 1-3.

25. E. Pollak and P. Talkner, Chaos, 2005, 15, 26116.

26. T. L. Bergman, A. Lavine, F. P. Incropera and D. P. DeWitt, Introduction to heat transfer, Wiley, 6th edn., 2011.

27. I. K. Hong and S. Lee, J. Ind. Eng. Chem., 2013, 19, 42-47.

28. M. R. Querry, Optical Constant of Minerals and Other Materials from the Millimeter to the Ultraviolet, Defense Technical Information Center Report, Department of Physics and Astronomy, University of Missouri-Kansas City, 1987. Retrieved from: https://apps.dtic.mil/docs /citations/ADA192210

29. J. D. J. Ingle and S. R. Crouch, Spectrochemical analysis, Old Tappan, NJ (US); Prentice Hall College Book Division, United States, 1988.

30. E. J. Wong, Modeling and Control of Rapid Cure in Polydimethylsiloxane (PDMS) for Microfluidic Device Applications, Ph.D. Thesis, Department of Mechanical Engineering, Massachusetts Institute of Technology, 2010.

31. K. Chenoweth, S. Cheung, A. C. T. Van Duin, W. A. Goddard and E. M. Kober, J. Am. Chem. Soc., 2005, 127, 7192-7202.

32. W. F. Manders and J. M. Bellama, J. Polym. Sci. Polym. Chem. Ed., 1985, 23, 351-357. 